\title{
An Efficient Load Balancing Algorithm Employing a Symmetric Balanced Incomplete Block Design
}

\author{
Okbin Lee ${ }^{1}$, Sangho Lee ${ }^{2}$, Seongyeol $\mathrm{Kim}^{3}$, and Ilyong Chung ${ }^{4 \star}$ \\ 1 Department of Computer Science, Chung-buk University, 361-763, Korea \\ lobin@mogent. net \\ 2 Department of Computer Science, Chung-buk University, 361-763, Korea \\ shlee@cbucc. chungbuk.ac.kr \\ 3 School of Computer and Information, Ulsan College, Ulsan, 682-090, Korea \\ kimsy@mail.ulsan-c.ac.kr \\ ${ }^{4}$ Dept. of Computer Science, Chosun University, Kwangju, 501-759, Korea \\ iyc@mail. chosun.ac. kr
}

\begin{abstract}
In order to maintain load balancing in distributed system, should obtain workload information from all the nodes on network. It requires $O\left(v^{2}\right)$ communication overhead, where $v$ is the number of nodes. In this paper, we present a new synchronous dynamic distributed load balancing algorithm on a $(v, k+1,1)$-configured network applying symmetric balanced incomplete block design, where $v=k^{2}+k+1$. Our algorithm needs only $O(v \sqrt{v})$ message overhead and each node receives workload information from all the nodes without redundancy. Therefore, load balancing is maintained since every link has the same amount of traffic for transferring workload information.
\end{abstract}

\section{Introduction}

In a distributed system it is likely that some processors heavily loaded while some are lightly loaded or idle. It is desirable that workload be balanced between these processors so that utilization of processors can be increased and response time can be reduced. A load balancing scheme [1]-[2] determines whether a task should be executed locally or by a remote processor. This decision can be made in centralized or distributed manner. In a distributed system, distributed manner is recommended. In order to make this decision, each node can be informed about workload information of other nodes. Also this information should be the latest because outdated information may cause an inconsistent view of system state from which adverse effect of load balancing. So disseminating load information may incur high link cost or significant communication traffic overhead.

In order to decrease communication overhead for obtaining workload information, the message is exchanged between adjacent nodes and local loadbalancing process is performed repeatedly. Then it makes the whole system be

\footnotetext{
* Corresponding Author (iyc@chosun.ac.kr)
} 
load-balancing [3]-[4]. It is accomplished by emplying CWA (Cube Walking Algorithm) on hypercube network, which utilizes workload information of all the nodes. This method requires $O\left(v^{2}\right)$ communication complexity and a communication path is $O\left(\log _{2} v\right)$. To reduce communication cost, flooding scheme is applied. However, the overlap of transmitting information occurs [5]-[6]. Based on SBN(Symmetric Broadcast Networks), communication patterns between nodes are constructed. It also needs $O\left(v^{2}\right)$ communication complexity for collecting workload information from all the nodes and a communication path is $O\left(\log _{2} v\right)$ $[7]-[8]$.

In this paper we present the network consisting of $v$ nodes and $v \times k$ links, each node of which is linked to $2 k$ nodes. On this network, each node sends workload information of $k$ nodes to $k$ adjacent nodes periodically. In other words, each node receives information from $k$ nodes and sends information to $k$ nodes. Also there is no redundancy in this received information. So, each node receives workload information for $k^{2}+k$ nodes with two round message interchange. Therefore load balancing in this algorithm is maintained so that every link has has same amount of traffic.

\section{About $(\boldsymbol{v}, \boldsymbol{k}, \boldsymbol{\lambda})$-Configuration}

Let $V=\{0,1, \ldots, v-1\}$ be a set of $v$ elements. Let $B=\left\{B_{0}, B_{1}, \ldots, B_{b-1}\right\}$ be a set of $b$ blocks, where $B_{i}$ is a subset of $V$ and $\left|B_{i}\right|=k$. For a finite incidence structure $\sigma=\{V, B\}$, if $\sigma$ satisfies following conditions, then it is a balanced incomplete block design(BIBD) [9], which is called a $(b, v, r, k, \lambda)$-configuration.

1. $B$ is a collection of $b k$-subsets of $V$ and this $k$-subsets are called the blocks.

2. Each element of $V$ is related with exactly $r$ of $b$ blocks.

3. Every two objects of $V$ appears simultaneously in exactly $\lambda$ of $b$ blocks.

4. $k<v$

For a $(b, v, r, k, \lambda)$-configuration, if it satisfies $k=r$ and $b=v$, then it is a symmetric balanced incomplete block design (SBIBD)[10] and it is called a $(v, k, \lambda)$ configuration. There are some relations among parameters $b, v, r, k, \lambda$ that are necessory conditions for existence of a corresponding $(b, v, r, k, \lambda)$-configuration.

1. In a $(b, v, r, k, \lambda)$-configuration, $b k=v r$ and $r(k-1)=\lambda(v-1)$

2. For a $(b, v, r, k, \lambda)$-configuration, $Q Q^{T}=(r-\lambda) I+\lambda J, I: v \times v$ identity matrix, $J: v \times v$ matrix in which all the entities are 1 's.

3. For a $(b, v, r, k, \lambda)$-configuration, $b \geq v$.

4. For a $(v, k, \lambda)$-configuration, every two blocs have exactly $\lambda$ objects in common .

As shown above, it is not true that there exists a BIBD or SBIBD for arbitrary set of parameters $b, v, r, k$ and $\lambda$. However there is no known sufficient condition on the existence of a certain $(b, v, r, k, \lambda)$-configuration or $(v, k, \lambda)$-configuration. 


\section{Generation of a $(v, k+1,1)$-Configuration}

We now present an algorithm to generate an incidence structure $\sigma=\{V, B\}$ satisfying the condition for a $(v, k+1,1)$-configuration in the case that $k$ is a prime number. This $(v, k+1,1)$-configuration is employed for constructing network topology below.

\subsection{Design of an Algorithm to Construct $(v, k+1,1)$-Configuration}

Algorithm 1 for Generating an incidence structure.

Incidence structure $T=\{V, B\}$, where $V=\{0,1, \ldots, v-1\}$, $B=\left\{B_{0}, B_{1}, \ldots, B_{b-1}\right\}$,

$\left|B_{i}\right|=k+1$. $B_{i, j}$ is the $j^{t h}$ element of $B_{i}$

1. Select a prime number $k$ and compute $v=k^{2}+k+1$.

2. Construct two incidence structures $X=\{V, C\}$ and $Y=\{V, D\}$.

a) $C_{i, j}, 0 \leq i, j \leq k$, has a value as following .

$$
-C_{i, j}=\left[\begin{array}{ll}
0 & \text { if } j=0 \\
t t=i \times k+j & \text { if } j \geq 1
\end{array}\right]
$$

b) $D_{i, j}, 0 \leq i \leq\left(k^{2}-1\right), 0 \leq j \leq k$, has a value as following .

$$
-D_{i, j}=\left[\begin{array}{lr}
C_{0, t}, t=\lfloor i / k\rfloor+1 & \text { if } j=0 \\
C_{j, t}, t=(i+(j-1) \times\lfloor i / k\rfloor) \bmod k+1 & \text { if } j \geq 1
\end{array}\right]
$$

3. Generate $Z=\{V, B\}$ from $X$ and $Y$.

$B_{i} \longleftarrow C_{i}$

$B_{i+k+1} \longleftarrow D_{i}$

The table below illustrates how to create $Z=\{V, B\}, V=\{0,1, \ldots, 6\}$. We now prove that this structure satisfies the conditions of a $(v, k+1,1)$ configuration.

Table 1. A set of blocks on $Z$ generated from Algorithm 1

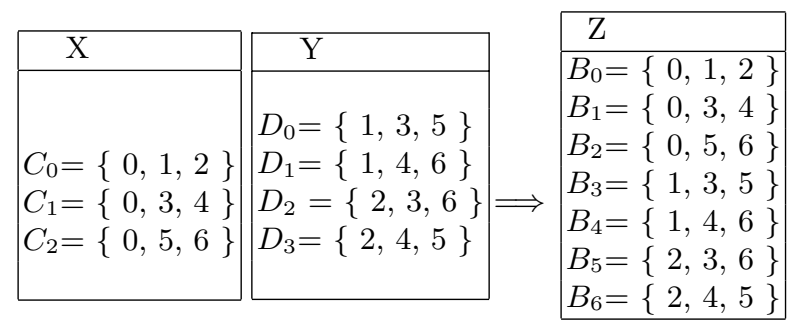


Definition 1: On incidence structure $Y$, Sector $S_{i}$ is the $i^{\text {th }}$ family of $k$ blocks, $D_{j} \in S_{i}, i=\lfloor j / k\rfloor$.

For example, If $k$ equals 3 , then $\lfloor 0 / k\rfloor=\lfloor 1 / k\rfloor=\lfloor 2 / k\rfloor=0$. So, $S_{0}=\left\{D_{0}, D_{1}, D_{2}\right\}$. There are $k$ sectors in $Y$.

Lemma 1: For two elements $D_{i 1, j 1}$ and $D_{i 2, j 2}, D_{i 1, j 1} \neq D_{i 2, j 2}$, if $j 1 \neq j 2$.

Proof: From Algorithm 1-2-(a), if $0<j \leq k, 0 \leq i \leq k$ then $C_{i, j}=i \times k+j$. This means if $j>0$ then all the elements are distinct. And as shown in Algorithm 1-2-(b), an element of $C_{j}$ is placed on $j^{\text {th }}$ element of a certain block of $Y$ if $D_{i, j}=C_{j, t}, t \neq 0$.

Lemma 2: For a sector consisting of $k$ blocks, the first element of each block has the same value and the other $k^{2}$ elements are equal to $V-C_{0}$.

Proof: In the case that $D_{i, 0}=C_{0,\lfloor i / k\rfloor+1}$, the first element of $k$ blocks on a sector have the same value. According to Algorithm 1-2-(b), $D_{i, j}=C_{j, t}, t=$ $(i+(j-1)\lfloor i / k\rfloor) \bmod k+1$. Since $k$ is a prime number, each element except the first element of each block is distinct and these distinct $k^{2}$ elements are equal to $V-C_{0}$.

Lemma 3: For incidence structure $Y, D_{a, j}=D_{b, j}, j \geq 1$, if $b=((a-c(j-1)) \bmod k+k(\lfloor a / k\rfloor+c)) \bmod k^{2}$.

Proof: From Algorithm 1-2-(b), $D_{a, j}=C_{j, t}$. We now prove that $D_{b, j}=C_{j, t}$. $t$ can be calculated from parameters $b, j$ below. Then $t$ obtained on this lemma is equal to that from Algorithm 1-2-(b). Therefore, $D_{a, j}=D_{b, j}$.

$$
\begin{aligned}
t= & (b+(j-1) \times\lfloor b / k\rfloor) \bmod k+1 \\
= & (((a-c(j-1)) \bmod k+k(\lfloor a / k\rfloor+c))+(j-1)\lfloor((a-c(j-1)) \bmod k+ \\
& k(\lfloor a / k\rfloor+c)) / k\rfloor) \bmod k+1 \\
= & (((a-c(j-1))+(j-1) \times(\lfloor a / k\rfloor+c) \bmod k+1 \\
= & (a+(j-1)\lfloor a / k\rfloor) \bmod k+1
\end{aligned}
$$

Here, if $D_{a, j}$ is in sector $S_{s}$ then $D_{b, j}$ is in $S_{(s+c) \bmod k}$. In case of $c \equiv$ $0(\bmod k)$, then $a=b$.

Lemma 4: Each element of $V$ appears in exactly $k+1$ times in $Z$.

Proof: According to Algorithm 1-2-(a), $C_{i, 0}=0$. Since $0 \leq i \leq k, 0$ appears $k+1$ times. The other $v-1$ elements, $V-\{0\}$, appear exactly once on X. From Lemma 3, each element of $C_{0, j}, 1 \leq j \leq k$, appears $k$ times in a sector of $Y$ and the rest $k^{2}$ elements appear once in every sector of Y. Therefore, each element appears $k+1$ times in Z.

Lemma 5: Any pair of elements of $V$ appears in exactly only once in $Z$.

Proof: The first element of $V$ makes a pair with all the other elements and this pair appears once by designing rule of incidence structure(see Algorithm 1-2-(a)). Each elements of $C_{0, j}, 1 \leq j \leq k$ makes a pair with $V-C_{0}$ elements and it also appears once proven by Lemma 3 . The rest $k^{2}$ elements are now considered. For an arbitrary pair $D_{a, j 1}=D_{a, j 2}, j 1, j 2 \geq 1$, in order to 
make the same pair on other block $D_{b}$, the two elements should be on the same block. According to Lemma 4 , if $j 1=j 2$, then they are located on $D_{b}$. However, this case does not occur since $j 1 \neq j 2$. Therefore, any pair of elements of $V$ appears in exactly only one time in $Z$.

Theorem 1: $Z$ designed by Algorithm 1 satisfies the conditions of a $(v, k+1,1)$ configuration.

Proof: $Z$ satisfied the conditions of the SBIBD by emplying Lemma 4 and Lemma 5.

\subsection{Design of Network Configuration}

In order to construct a network topology which have minimum link cost and traffic overhead, we imported $(v, k+1,1)$-configuration. An incidence structure $Z=\{V, B\}$ satifies the conditions for a $(v, k+1,1)$-configuration and $M$ be a binary incidence matrix of $Z$. Then this matrix $M$ can be transformed to an adjacent matrix of a graph $G=\{V, E\}$. Based on this idea, network topology can be designed as follows.

Algorithm 2 for Design of Network Configuration.

1. Create an incidence structure $Z=\{V, B\}$ by Algoritm 1 .

2. Generate $L=\{V, E\}$ from $Z$ by exchanging blocks so that every block $i$ includes object $i$.

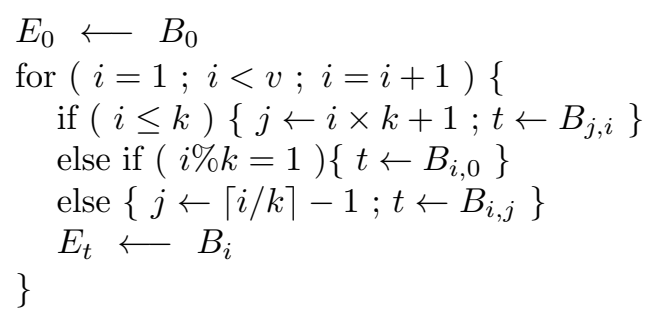

3. Create an adjacent matrix $A=\left(a_{i j}\right)$ for graph $G$ from $L$, where $\mathrm{G}$ is a network topology containing $v$ processors.

$$
a_{i j}=\left[\begin{array}{ll}
1 & \text { if } i \neq j, \text { and if } i \in E_{j} \text { or } j \in E_{i} \\
0 & \text { otherwise }
\end{array}\right]
$$

$G$ has $v$ nodes since $G$ is created from $(v, k+1,1)$-configuration. Each block $L[i]$ is composed of $k+1$ elements and $i$ is the one of them. Each node obtains $2 k$ links from Step 3 of Algorithm 2. So, G become a 2k-regular graph. therefore there are $(2 k \times v) / 2=v k$ links in $G$. Given $Z=\{V, B\}$ described on Fig.1, performance of Algorithm 2 is shown on Table 2 and Fig.1. 
Table 2. Blocks of $L$ generated from $Z$ of Table 1

\begin{tabular}{|c|}
\hline $\mathrm{L}$ \\
\hline$E_{0}=\{0,1,2\}$ \\
$E_{1}=\{1,3,5\}$ \\
$E_{2}=\{2,3,6\}$ \\
$E_{3}=\{0,3,4\}$ \\
$E_{4}=\{1,4,6\}$ \\
$E_{5}=\{2,4,5\}$ \\
$E_{6}=\{0,5,6\}$ \\
\hline
\end{tabular}

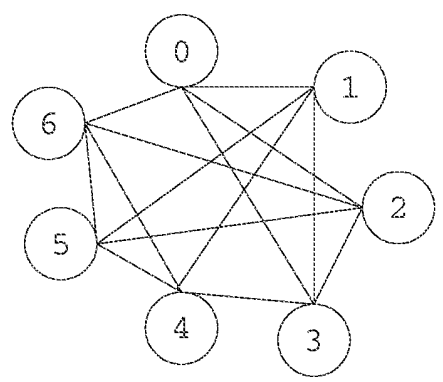

Fig. 1. (7,3,1)-configured network obtained from $L$

\section{Design of an Efficient Load Balancing Algorithm on $(\mathrm{v}, \mathrm{k}+1,1)$-Configured Networks}

An efficient load balancing algorithm is now constructed on $(\mathrm{v}, \mathrm{k}+1,1)$-configured networks generated by Algorithm 2 .

Definition 2 : Construct two sets $S_{i}$ and $R_{i}$ consisting of adjacent $k$ nodes, where $S_{i}$ is a set of nodes to which node $i$ sends workload information and $R_{i}$ is a set of nodes to receive $i$ 's workload information.

$S_{i}=\left\{v \mid v \in E_{i}-i\right\}$

$R_{i}=\left\{v \mid i \in E_{v}\right.$ and $\left.i \neq v\right\}$

Definition 3 : Generate two sets $S F_{i}$ and $R F_{i}$, where $S F_{i}(j)$ is a set of workload information for $i$ 's adjacent nodes transmitted from node $i$ to node $j$ at time $T_{2 t}$ and $R F_{i}(j)$ is $i$ 's workload information transmitted from node $i$ to node $j$ at time $T_{2 t+1}$.

$S F_{i}=\left\{S F_{i}(j) \mid j \in S_{i}, S F_{i}(j)=\left\{E_{i}-\{j\}\right\}\right.$. $R F_{i}=\left\{R F_{i}(j) \mid j \in R_{i}, R F_{i}(j)=i\right\}$.

Algorithm 3 for Construction of an Efficient Load Balancing Algorithm.

1. Node $i$ sends a set of workload information $S F_{i}(j)$ to node $j \in S_{i}$ at $T_{2 t}$ and renews a table of workload information. 
2. Node $i$ sends a set of workload information $R F_{i}(j)$ to node $j \in R_{i}$ at $T_{2 t+1}$ and renews a table of workload information.

3. Repeat the first step.

The following table indicates that node $i$ sends workload information $S F_{i}(j)$ and $R F_{i}(j)$ to node $j$ at times $T_{2 t}$ and $T_{2 t+1}$, respectively. So every node can obtain workload information for all the nodes at $T_{2 t+2}$ and this fact is proven in Theorem 2.

Table 3. Two steps for sending workload information from Node $i$

\begin{tabular}{|c|l|c|}
\hline Node ID & \multicolumn{1}{|c|}{$T_{2 t}$} & $T_{2 t+1}$ \\
\hline \hline 0 & $S F_{0}(1)=\{0,2\} S F_{0}(2)=\{0,1\}$ & $R F_{0}(3)=\{0\} R F_{0}(6)=\{0\}$ \\
\hline 1 & $S F_{1}(3)=\{1,5\} S F_{1}(5)=\{1,3\}$ & $R F_{1}(0)=\{1\} R F_{1}(4)=\{1\}$ \\
\hline 2 & $S F_{2}(3)=\{2,6\} S F_{2}(6)=\{2,3\}$ & $R F_{2}(0)=\{2\} R F_{2}(5)=\{2\}$ \\
\hline 3 & $S F_{3}(0)=\{3,4\} S F_{3}(4)=\{3,0\}$ & $R F_{3}(1)=\{3\} R F_{3}(2)=\{3\}$ \\
\hline 4 & $S F_{4}(1)=\{4,6\} S F_{4}(6)=\{4,1\}$ & $R F_{4}(3)=\{4\} R F_{4}(5)=\left\{\begin{array}{l}4 \\
4\end{array}\right.$ \\
\hline 5 & $S F_{5}(2)=\{5,4\} S F_{5}(4)=\{5,2\}$ & $R F_{5}(1)=\{5\} R F_{5}(6)=\{5\}$ \\
\hline 6 & $S F_{6}(0)=\{6,5\} S F_{6}(5)=\{6,0\}$ & $R F_{6}(2)=\{6\} R F_{6}(4)=\{6\}$ \\
\hline
\end{tabular}

Theorem 2: According to Algorithm 3, every node obtains workload information for all the nodes at $T_{2 t+2}$.

Proof: At $T_{2 t}$, node $i$ sends workload information for $S F_{i}(j)$ to node $j$. On an aritrary pair $\left(S F_{i 1}(j), S F_{i 2}(j)\right), i 1 \neq i 2$, intersection of these sets is empty since on $(\mathrm{v}, \mathrm{k}+1,1)$-configuration, every two objects appears simultaneously in exactly one of $v$ blocks and node $j$ is an element of $S_{i 1}$ and $S_{i 2}$, respectively. So node $j$ obtains workload information for $k^{2}$ nodes. And at $T_{2 t+1}$, node $i$ transmits its workload information to node $j$ by Algorithm 3-2. Then, node $j$ receives $k$ workload information. Therefore, node $j$ receives workload information for $k^{2}+k$ nodes at $T_{2 t+2}$.

\section{Conclusion}

In order for the system to increase utilization and to reduce response time, workload should be balanced. In this paper, we present an efficient load balancing algorithm on $(v, k+1,1)$-configured networks consisting of $v$ nodes and $v k$ links. Our algorithm needs only $O(v \sqrt{v})$ message overhead and each node receives workload information from all the nodes without redundancy and load balancing is maintained so that every link has same amount of traffic for transferring workload information. 


\section{References}

1. M. Willebeek-Lemair and A. P. Reeves, Strategies for dynamic load-balancing on highly parallel computers, IEEE Transactions on Parallel and Distributed Systems, vol. 4, no. 9, pp. 979-993, 1993.

2. B.A. Shirazi, Scheduling and load balancing in parallel and distributed systems, IEEE Computer Society Press, 1995.

3. S. Hosseini, B. Litow, M. Malkawi, Analysis of a graph coloring based distributed load balancing algorithm, Journal of Parallel and Distributed Computing, vol. 10, no. 2, pp. 160-166, 1990.

4. C.Hui, S.Chanson, Hydrodynamic Load Balancing, IEEE Transactions on Parallel and Distributed System, vol. 10, no. 11, pp. 1118-1137, 1999.

5. K. Nam, J. Seo, Synchronous Load balancing in Hypercube Multicomputers with Faulty Nodes, Journal of Parallel and Distributed Computing, vol. 58, pp. 26-43, 1999.

6. H. Rim, J. Jang, Method for Maximal Utilization of Idle links for Fast Load Balancing, Journal of Korea Information Processing Society, vol. 28, no. 12, 2001.

7. S. Das, D. Harvey, and R. Biswas, Adaptive Load-Balancing Algorithms Using Symmetric Broadcast Networks, NASA Ames Research Center, TR NAS-97-014, May 1997.

8. S. Das, D. Harvey, and R. Biswas, Parallel Processing of Adaptive Meshes with Load Balancing, IEEE Transactions on Parallel and Distributed Systems, vol. 12, no. $12,2001$.

9. C.L.Liu, Block Designs in Introduction to Combinatorial Mathematics, McGrawHill, pp. 359-383, 1968.

10. I. Chung, W. Choi, Y. Kim, M. Lee, The Design of conference key distribution system employing a symmetric balanced incomplete block design, Information Processing Letters, vol. 81, no. 6, pp. 313-318, 2002.3. 